ارزيابى عوامل رونويسى در يك مطالعه ترانسكرييتوم در نخود (Cicer arietinum L.)

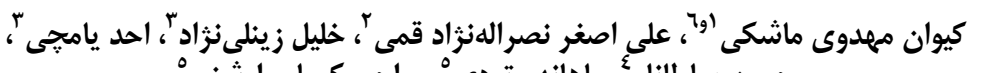

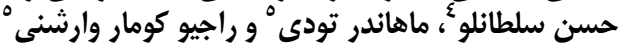

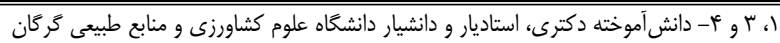

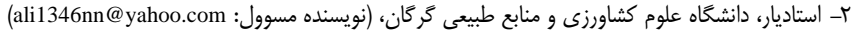

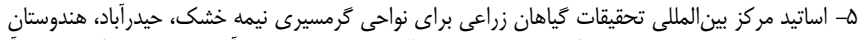

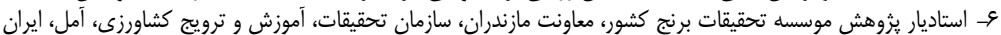
تاريخ دريافت:

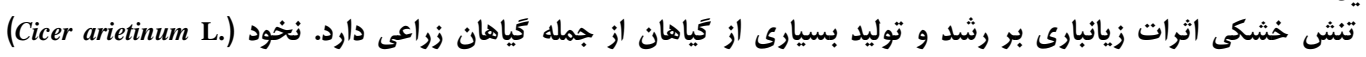

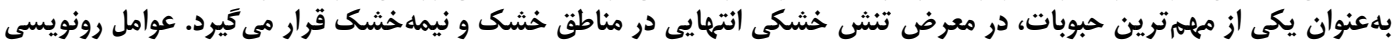

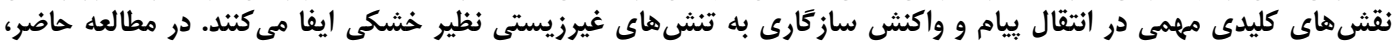

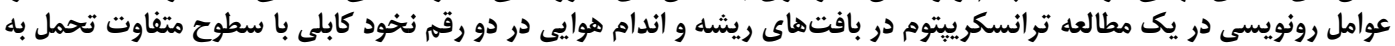

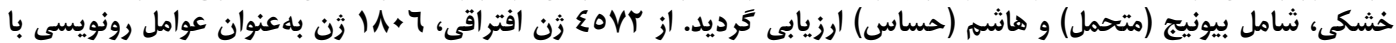

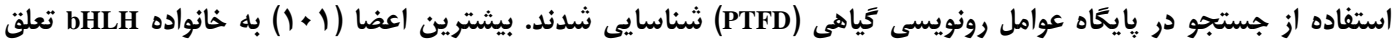

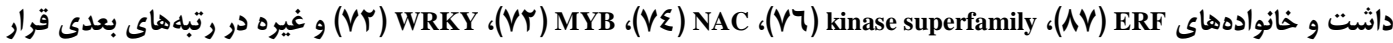

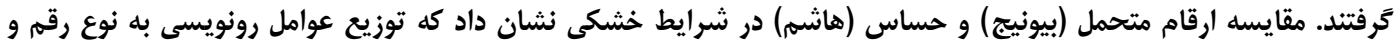

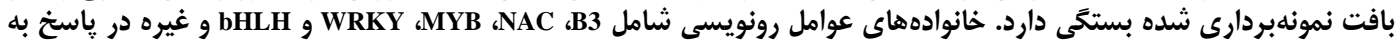

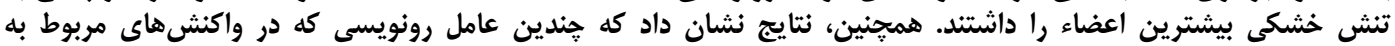

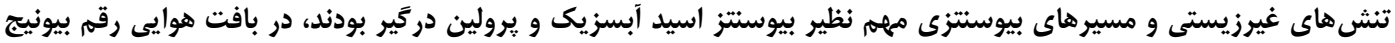

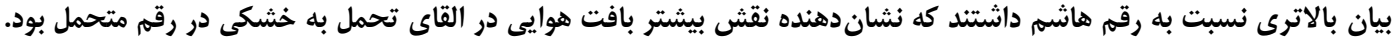

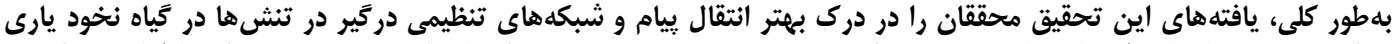

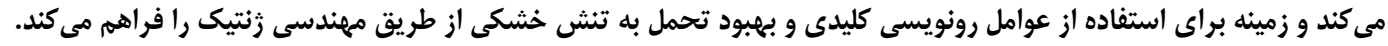

وازههاى كليدى: نخود، تنش خشكى، ترانسكرييتوم، انتقال ييام، عوامل رونويسى

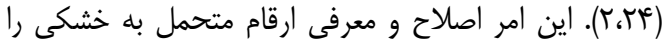

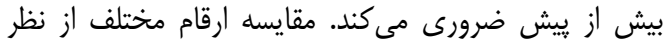

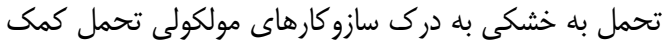

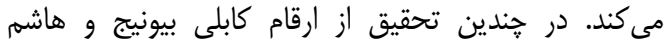

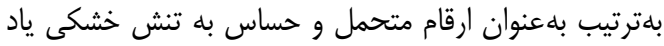

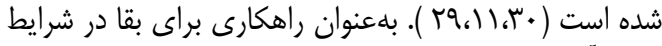

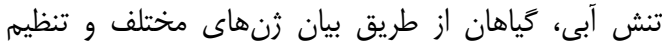

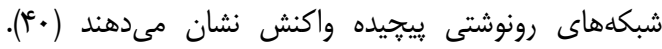

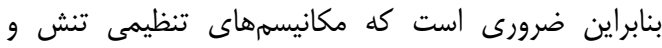

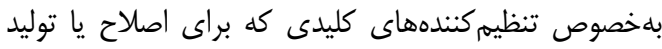

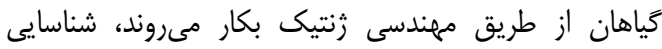

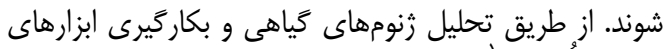

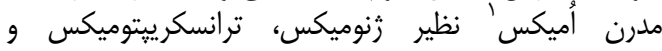

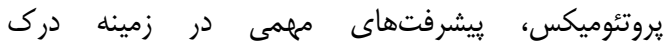

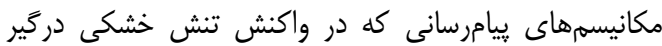

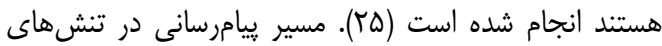

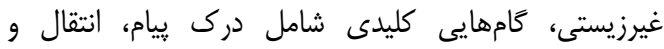

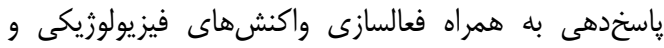

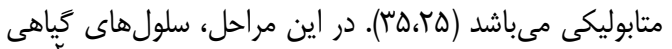

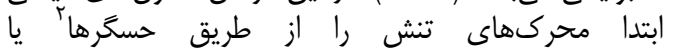

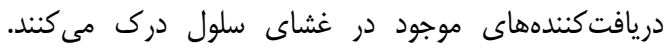

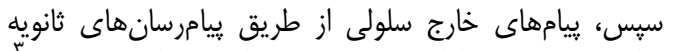

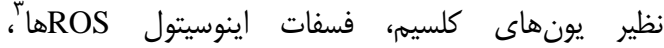
نوكلئوتيدهاى حلقوى (cAMP و cAMP)، قندها و غيره به
نخود (Cicer arietinum L. بلهنوان يكى از لكَومهاى

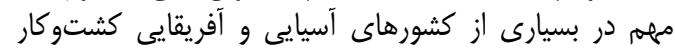

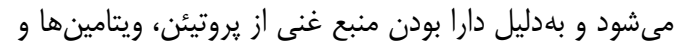

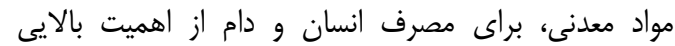

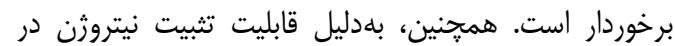

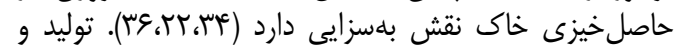

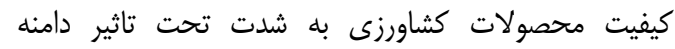

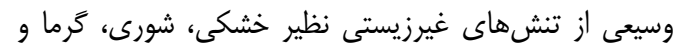

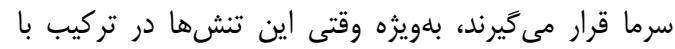

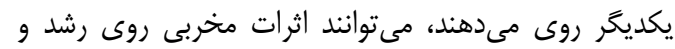

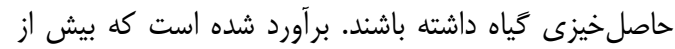

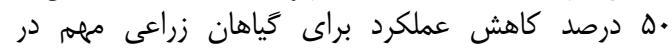

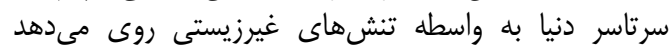

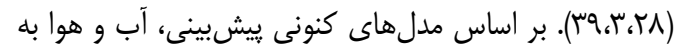

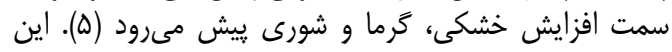

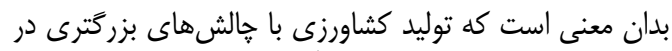

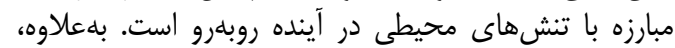

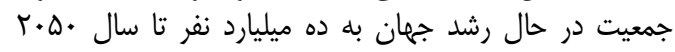

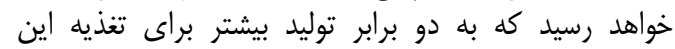

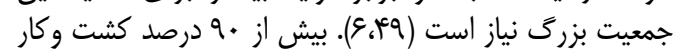

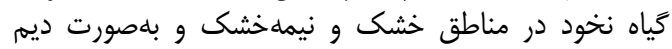

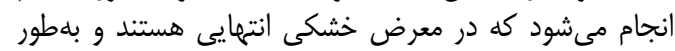

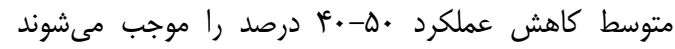




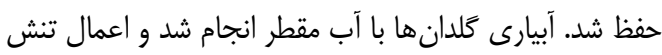

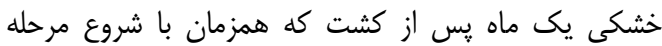

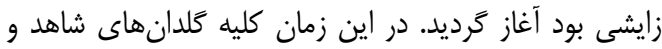

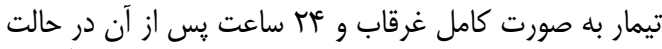

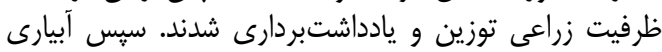

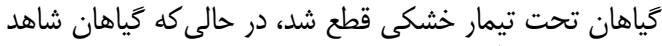

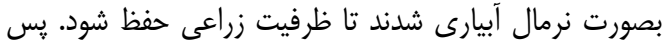

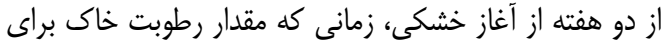

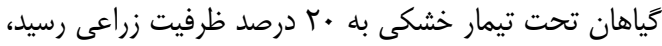

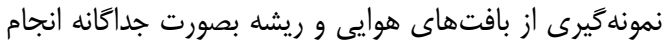

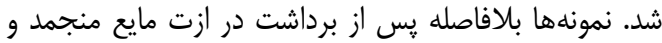

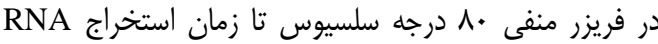
نخمبدارى شدند.

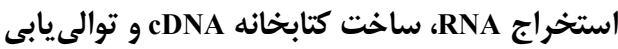

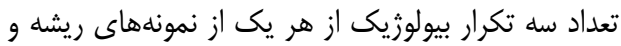

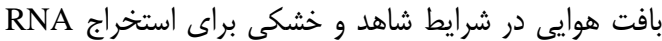

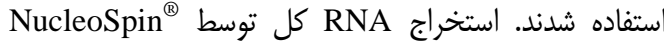
MACHEREY-NAGEL GmbH ) RNA Plant kit (and Co. KG, Germany, 2013 سازنده انجام شد. كميت و كيفيت RNA استخراجى با

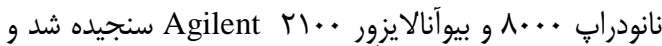

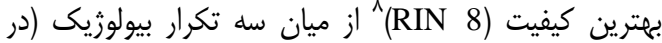

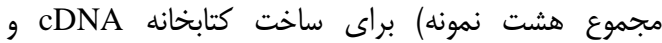
توالى يابى استفاده كرديد. ساخت كتابخانه cDNA با استفاده أز از

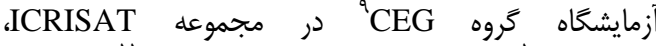

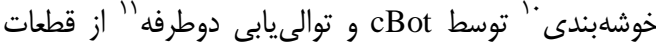
شف Ir جفت بازى با دستخاه Illumina HiSeq2500 انجام

نقشهيابى با استفاده از زنوم مرجع، آناليز بيان زن و و

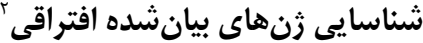

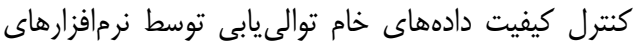
FastQC

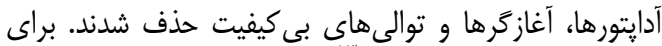

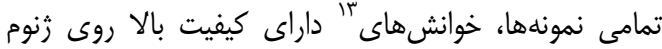

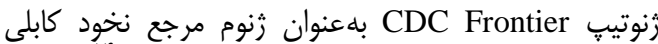

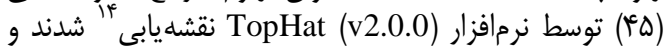

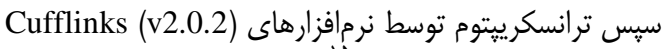

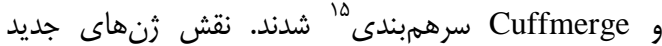

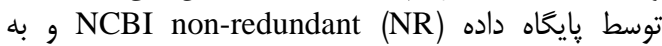

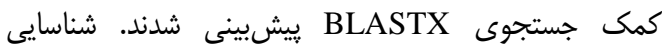
ثن هاى داراى بيان افتراقى بين نمونهها با استفاده از نرمافزار

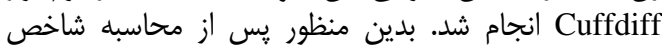

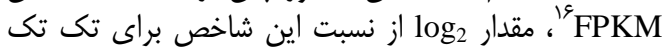

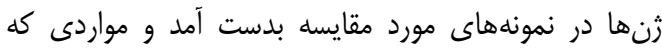
بزر كتر يا مساوى دو و داراى معيار معنى دارى ثنهاى افتراقى لحاظ شدند.
موارد درون سلولى تبديل مىشوند. متعاقباً، اين يِيامرسان هاى رياى

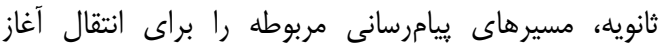

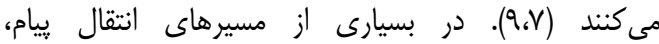

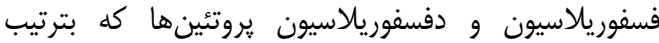

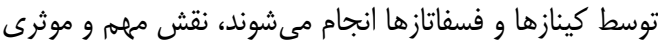

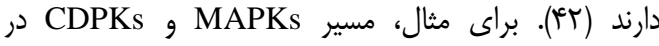

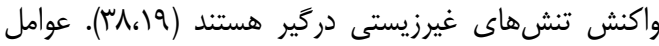

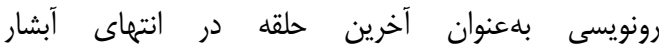

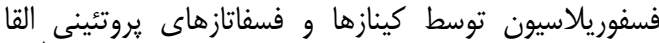

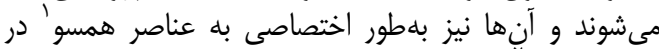

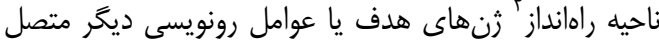

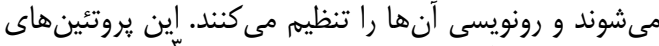

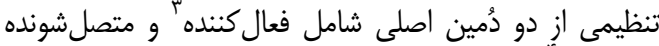

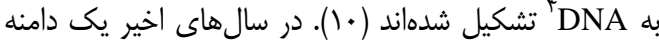

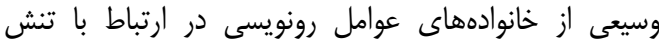

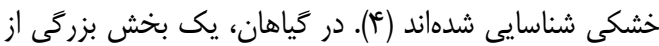

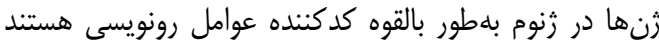

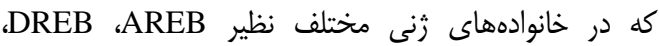

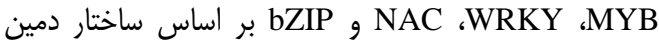

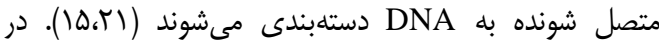

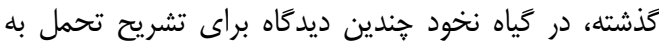

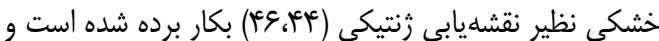

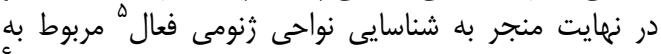

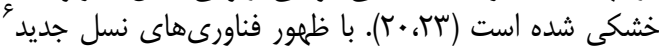

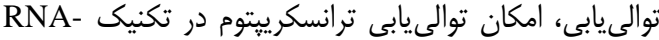

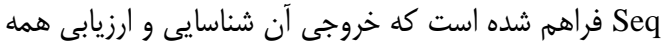

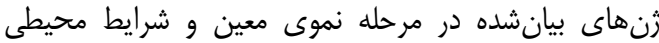

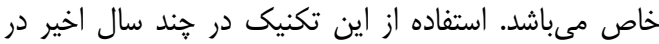

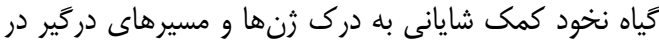

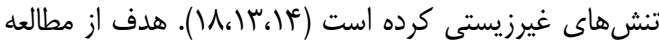

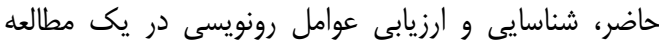

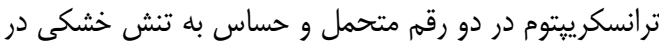
كياه نخود مىباشد.

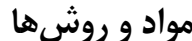

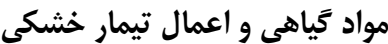

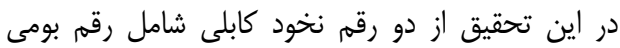

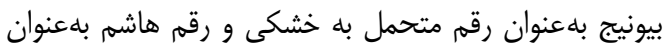

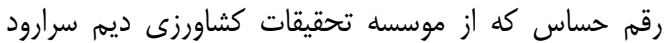

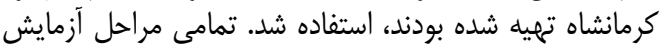

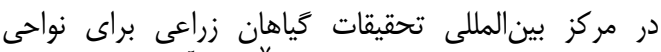

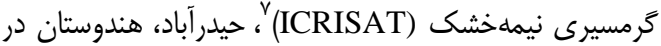

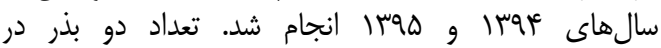

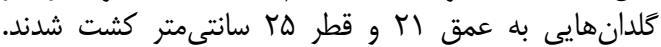

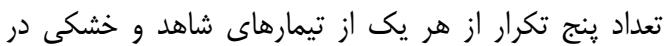

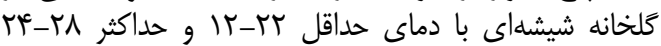

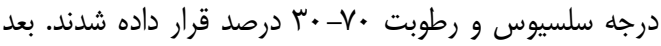
از هشت روز از جوانهزنى تنها يك گياه سالم در هر كلدان

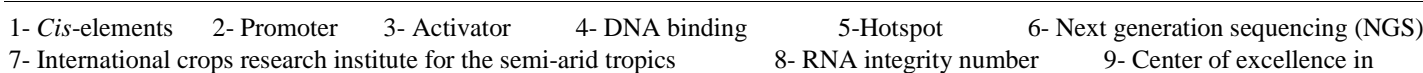
$\begin{array}{lllll}\text { 7- International crops research institute for the semi-arid tropics } & \text { 8- RNA integrity number } & \text { 9- Center of excellence in } \\ \text { genomics } & \text { 10- Clustering } & \text { 11- Paired-end } & \text { 12- Differentially expressed genes (DEG) } & \text { 13-Reads }\end{array}$ 15- Assembly 16- Fragments per kilobase of transcript per million mapped reads 
يك مطالعه ترانسكرييتوم روى ريشه نخود نوع دسى تحت

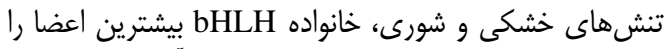

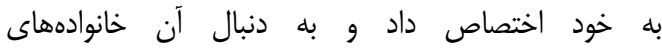

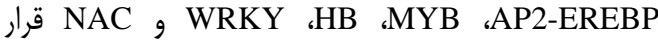

داشتند (If).

ارزيابى عوامل رونويسى در ريشه و وافئ بافت هوايى در مقايسه بين دو رقمى در شرايط خشيكى رونى

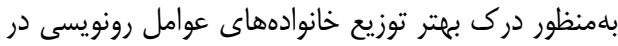

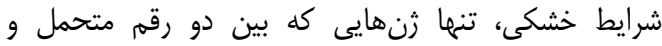

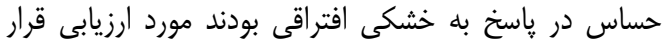

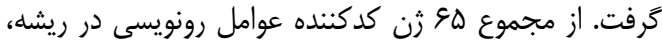

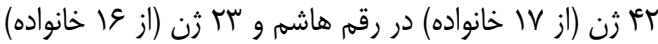

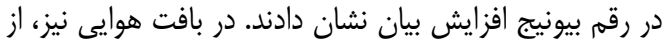

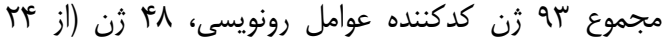

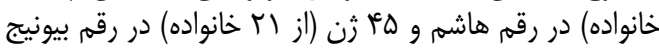

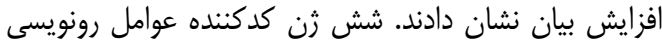

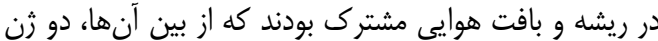

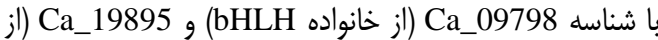
خانواده MYB-related در ريشه رقمه هاشم بيان بيشترى از

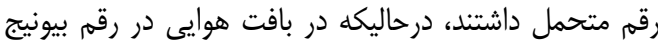
بيان بيشترى نسبت به رقم حساس داشتند.
شنتاسـايى عوامـل رونويسـى از ميـان زنهــاى بيـانى

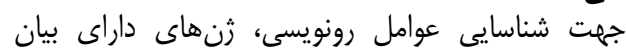

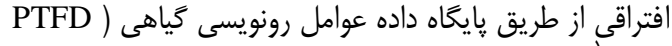

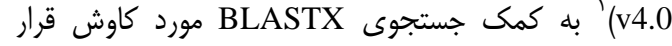

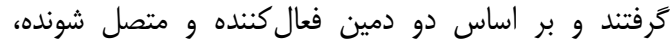

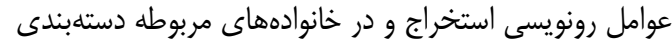

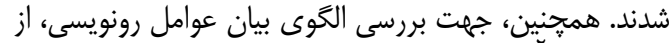
نقشه دمايى بّهي كمك نرمافزار MeV v4.8.1 استفاده شد.

\section{ت ت ايج و بحث}

ارزيابى عوامل رونويسى در كل زنه نهاى بيانى افتراقى

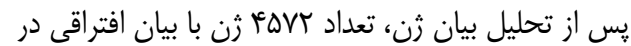

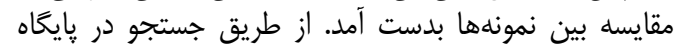

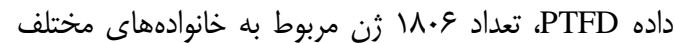

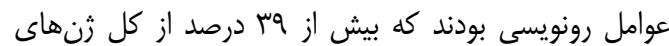

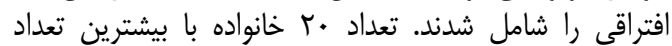

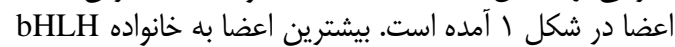

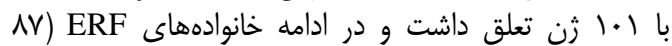

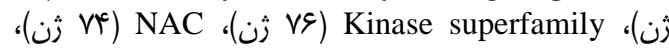
(ז) MYB

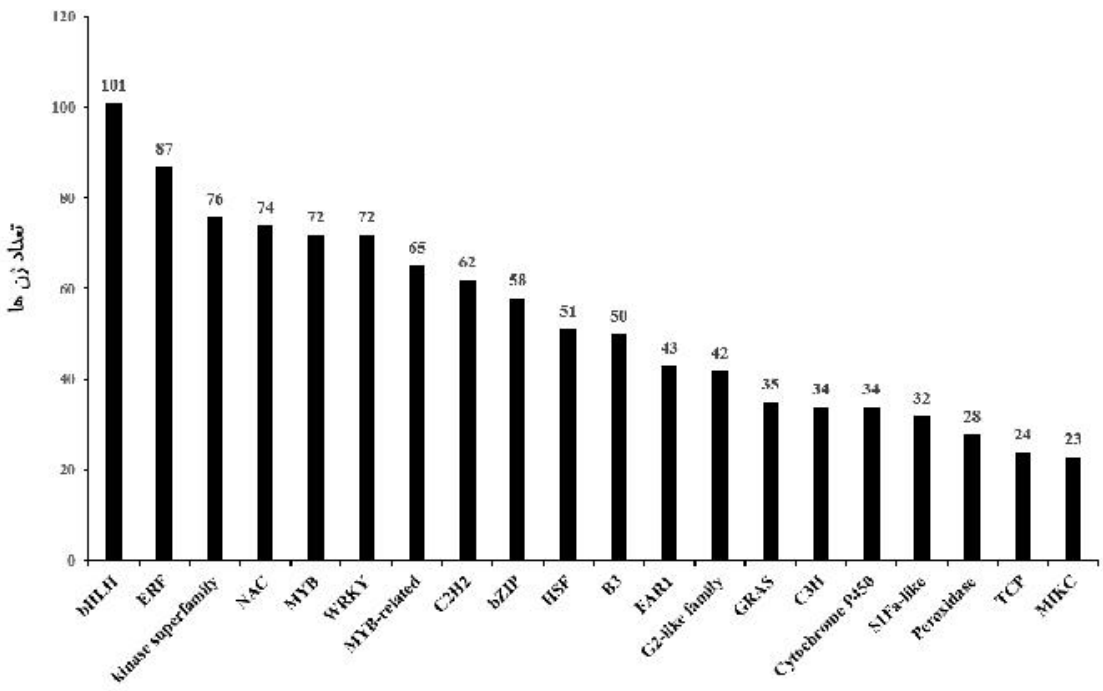

شكل ا- توزيع •r خانواده عوامل رونويسى با بيشترين تعداد عضو از ميان بهأ زن بيانى افتراقى.

Figure 1. Distribution of top 20 transcription factor families among the 4572 differentially expressed genes 
(ب)

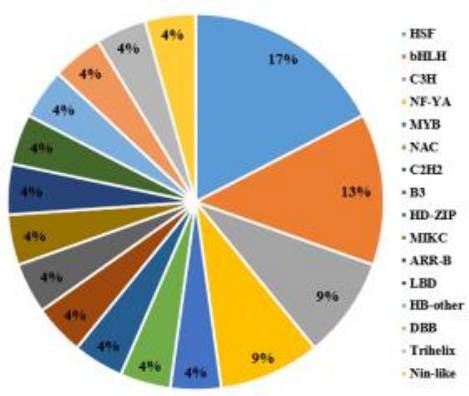

(ت)

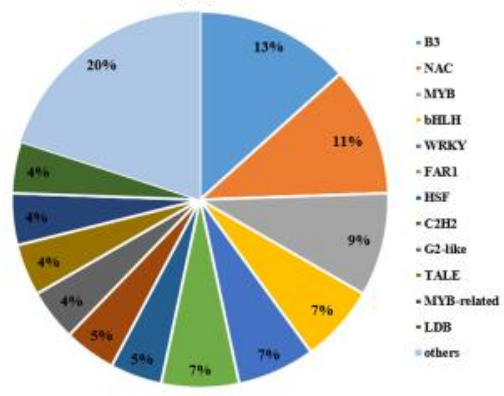

(الف)

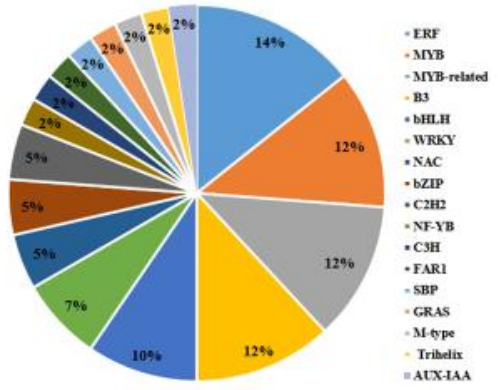

(ب)

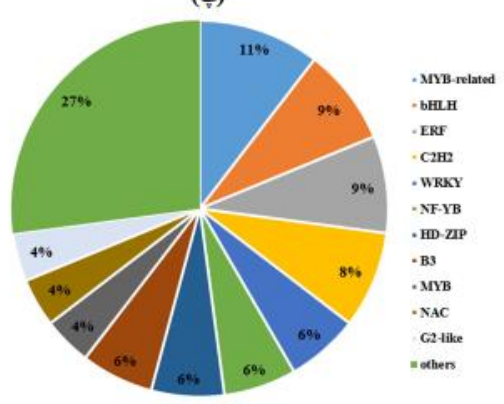

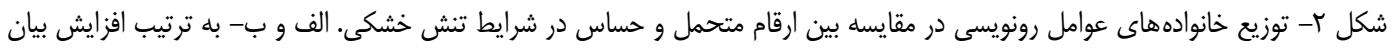

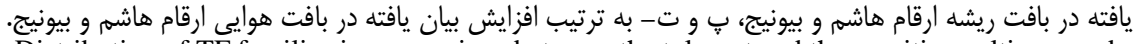

Figure 2. Distribution of TF families in comparison between the tolerant and the sensitive cultivars under drought stress. A and B- Up-regulated in the roots of Hashem and Bivanij, respectively. C and D- Up-regulated in the shoots of Hashem and Bivanij, respectively.

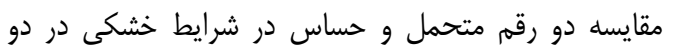

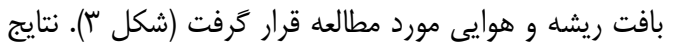

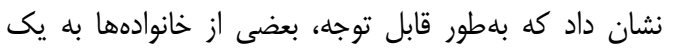

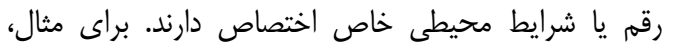

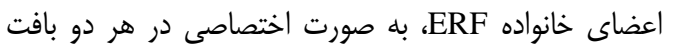

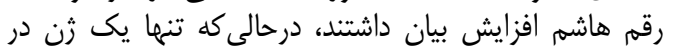

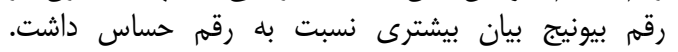

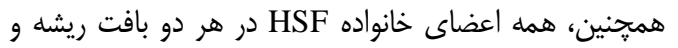

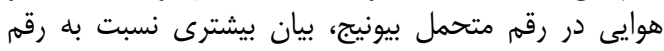

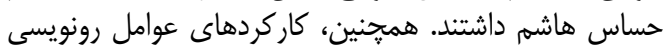

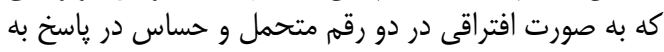

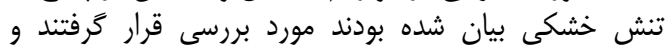

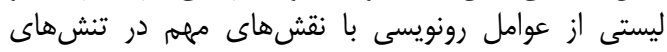

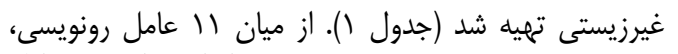

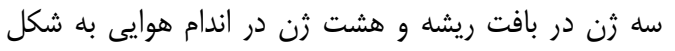

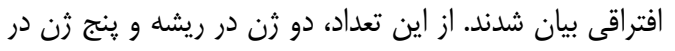

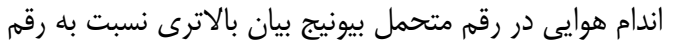

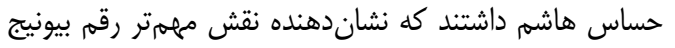

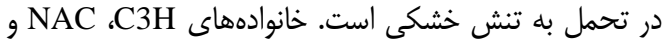
MYB-related
در مقايسه دو رقمم متحمل و حساس در شرايط خشكى در

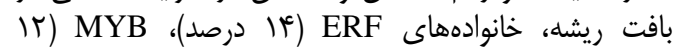

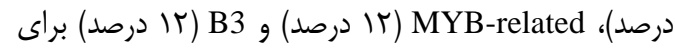

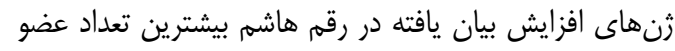

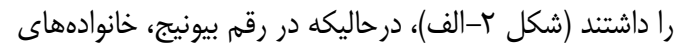
bHLH (Y) BSF به NF-YA

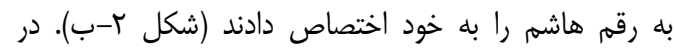
بافت هوايى نيز، خانوادههاى MYB-related (1) (1 دروصد)، bHLH

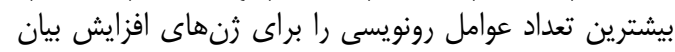

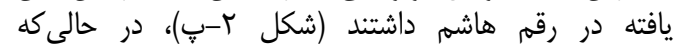

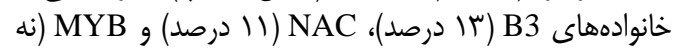

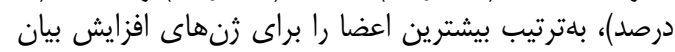

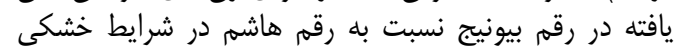

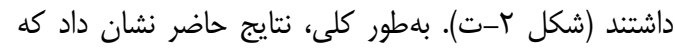

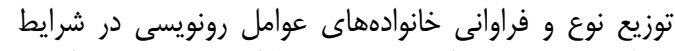

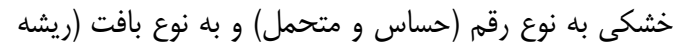

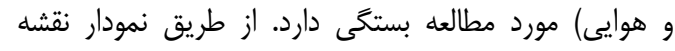

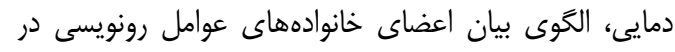


مسير بيوسنتز كاروتنوئيد كه منجر به سنتز هورمون اسيد

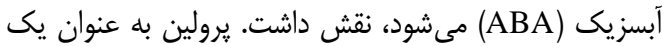

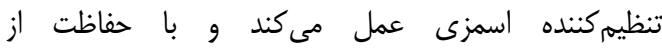

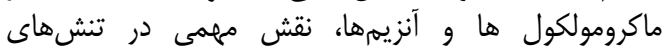

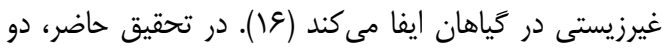
زن عوامل رونويسى B3 و مAC بودند در مراحل بيوستنز يرولين نقش داشتند و بيان هر دو زئن در اندام هوايى رقمم بيونيج

$$
\text { بيشتر از رقم هاشم بود. }
$$

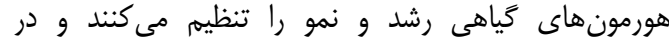

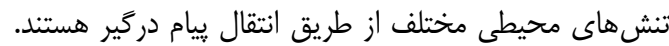

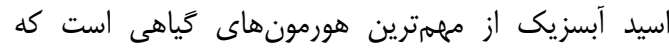

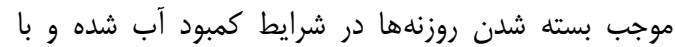

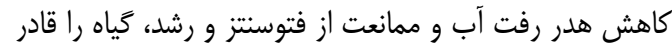

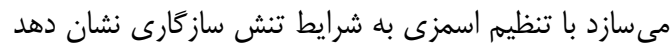

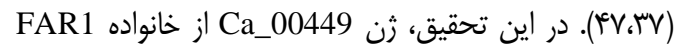

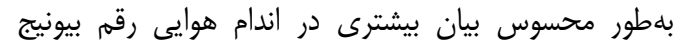

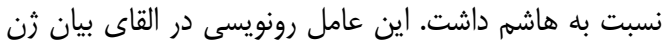

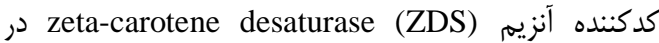

جدول ا- ليستى از عوامل رونويسى افتراقى بين دو رقم مورد مطالعه در شرايط خشكى كه داراى نقشهاى كاركردى كليدى در ارتباط با تنش هستند

Table 1. List of differentially TFs between the cultivars in drought stress response which have key functional roles

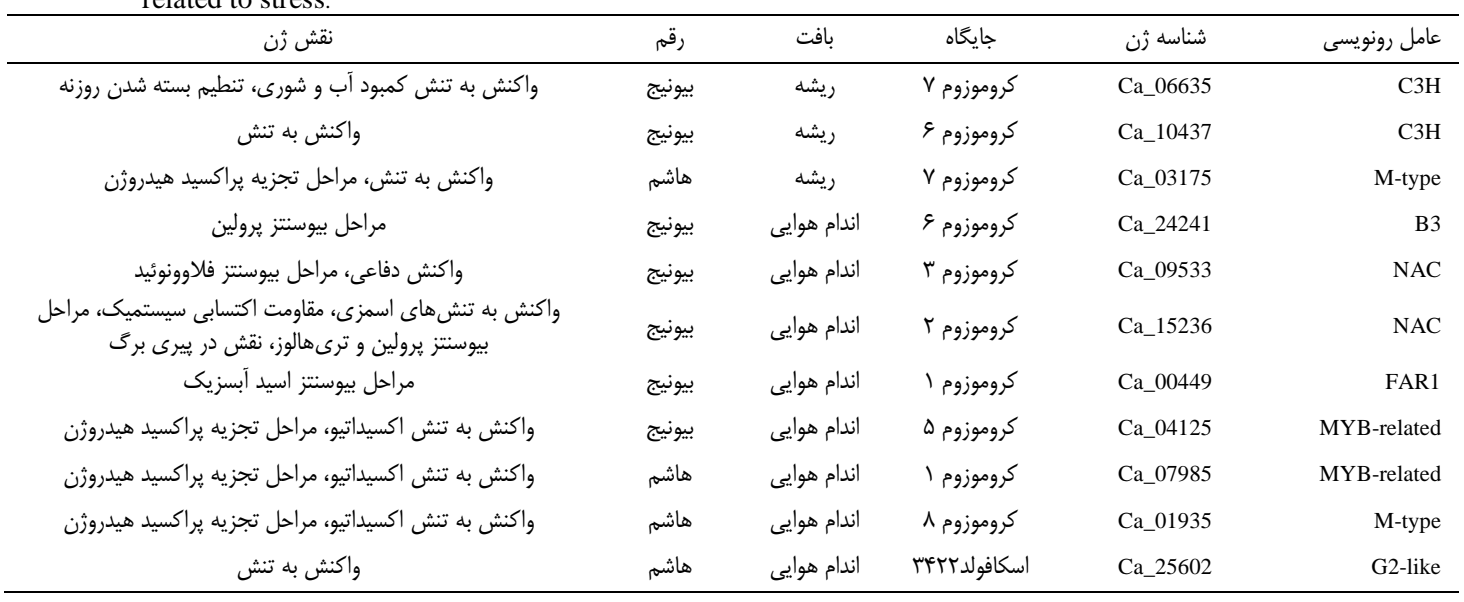

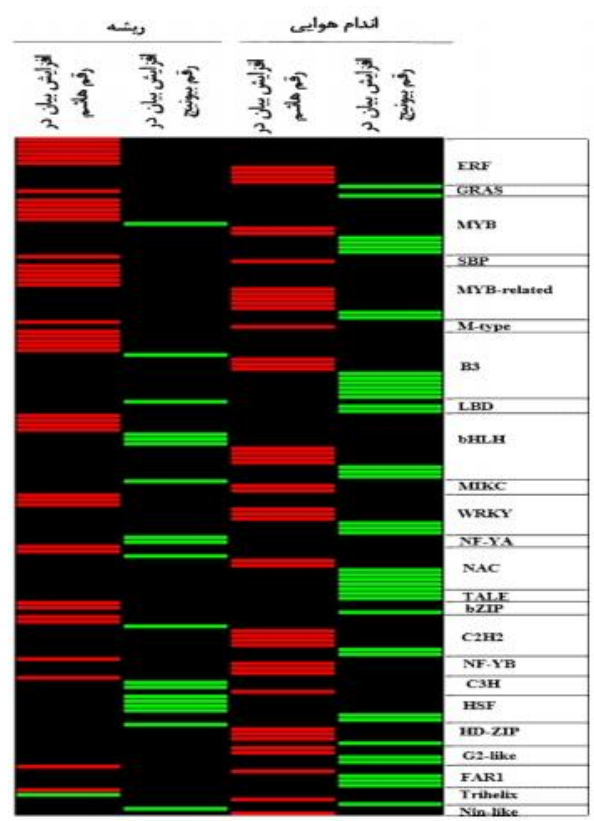

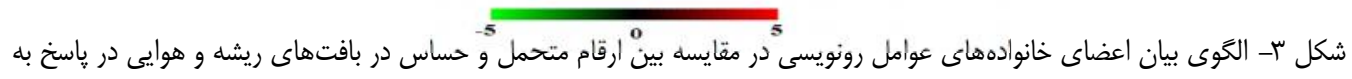

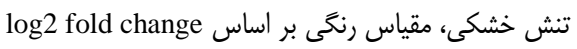

Figure 3. Expression patterns of TF family members in comparison of the tolerant and the sensitive genotypes in drought stress response, Color scale are based on $\log 2$ fold change 
خشكى نظير COR ERD RD و ت غيره افزايش نشان داد و

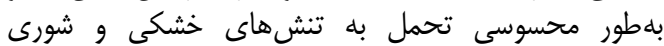

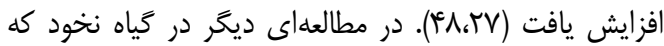

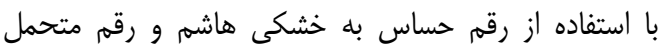
انجام شد، عامل رونويسى ILC482

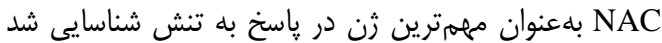

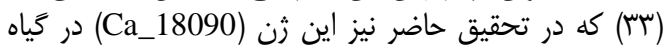

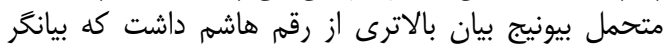

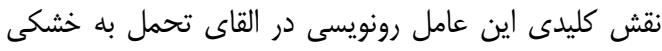

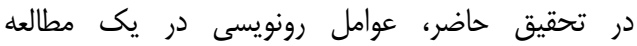

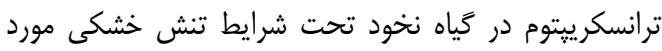

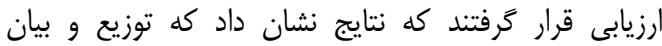

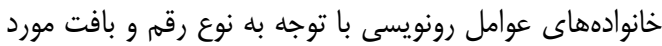

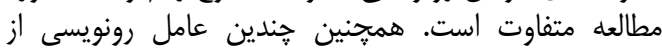

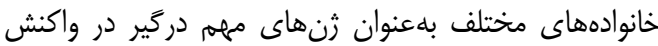

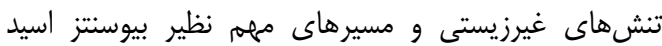

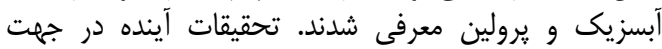

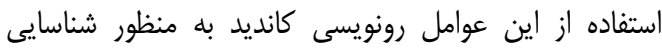

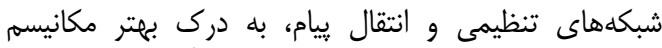

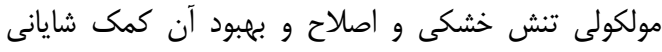
خواهد كرد.
مطالعات كذشته انواع متنوعى از عوامل رونويسى را كه به

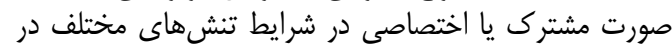

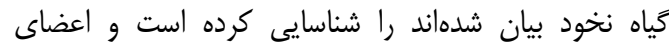
خانوادههاى WHF ، AP2/EREBP ،inc-finger

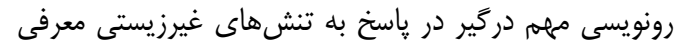

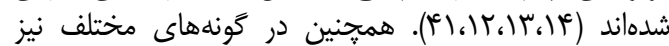
جندين عضو از عوامل رونويسى MYB و و

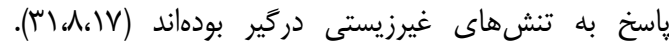

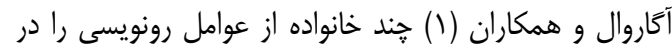

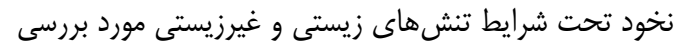

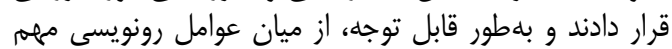

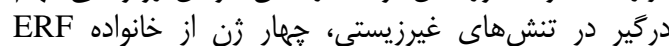
و Ca_Ca_Ca_23799 ، Ca_15031) دو ثن از خانواده Ca_25811) G2-like و مو Ca_Ca_25602) با

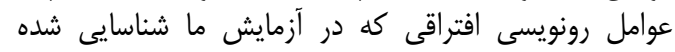

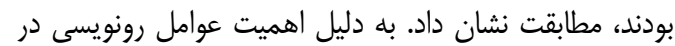

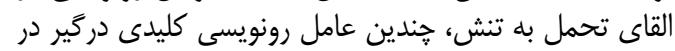

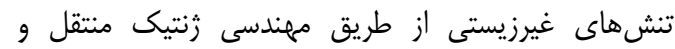

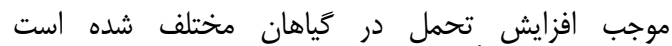

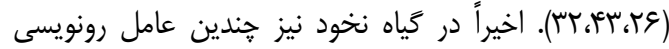

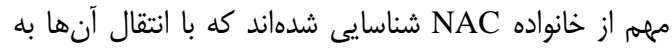

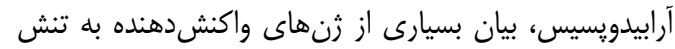

1. Agarwal, G., Genome-wide dissection of AP2/ERF and HSP90 gene families in five legumes and expression profiles in chickpea and pigeonpea. Plant Biotechnology Journal, 14: 1563-1577.

2. Ahmad, F., P. Gaur and J. Croser. 2005. Chickpea (Cicer arietinum L.). Genetic Resources, Chromosome Engineering and Crop Improvement-Grain Legumes, 1: 185-214.

3. Ahuja, I., R.C. de Vos, A.M. Bones and R.D. Hall. 2010. Plant molecular stress responses face climate change. Trends in Plant Science, 15: 664-674.

4. Anbazhagan, K., P. Bhatnagar-Mathur, V. Vadez, S.R. Dumbala, P.K. Kishor and K.K. Sharma. 2015. DREB1A overexpression in transgenic chickpea alters key traits influencing plant water budget across water regimes. Plant Cell Reports, 34: 199-210.

5. Bates, B. 2009. Climate Change and Water: IPCC technical paper VIWorld Health Organization, 101 p.

6. Bengtsson, M., Y. Shen and T. Oki. 2006. A SRES-based gridded global population dataset for 19902100. Population and Environment, 28: 113-131.

7. Bhargava, S. and K. Sawant. 2013. Drought stress adaptation: metabolic adjustment and regulation of gene expression. Plant Breeding, 132: 21-32.

8. Bhattacharjee, A. and M. Jain. 2013. Transcription factor mediated abiotic stress signaling in rice. Plant Stress, 7: 16-25.

9. Chaves, M., J. Flexas and C. Pinheiro. 2009. Photosynthesis under drought and salt stress: regulation mechanisms from whole plant to cell. Annals of Botany, 103: 551-560.

10.Danquah, A., A. de Zelicourt, J. Colcombet and H. Hirt. 2014. The role of ABA and MAPK signaling pathways in plant abiotic stress responses. Biotechnology Advances, 32: 40-52.

11.Farshadfar, E. and J. Javadinia. 2011. Evaluation of chickpea (Cicer arietinum L.) genotypes for drought tolerance. Seed and Plant Improvement Journal, 27(1): 517-537 (In Persian).

12. Gao, W.R., X.S. Wang, Q.Y. Liu, H. Peng, C. Chen, J.G. Li, J.S. Zhang, S.N. Hu and H. Ma. 2008. Comparative analysis of ESTs in response to drought stress in chickpea ( $C$. arietinum L.). Biochemical and Biophysical Research Communications, 376: 578-583. 
13.Garg, R., A. Bhattacharjee and M. Jain. 2015. Genome-scale transcriptomic insights into molecular aspects of abiotic stress responses in chickpea. Plant Molecular Biology Reporter, 33: 388-400.

14.Garg, R., R. Shankar, B. Thakkar, H. Kudapa, L. Krishnamurthy and N. Mantri. 2016. Transcriptome analyses reveal genotype-and developmental stage-specific molecular responses to drought and salinity stresses in chickpea. Scientific Reports, 6: 19228.

15. Golldack, D., I. Lüking and O. Yang. 2011. Plant tolerance to drought and salinity: stress regulating transcription factors and their functional significance in the cellular transcriptional network. Plant Cell Reports, 30: 1383-1391.

16. Hayat, S., Q. Hayat, M.N. Alyemeni, A.S. Wani, J. Pichtel and A. Ahmad. 2012. Role of proline under changing environments: a review. Plant Signaling \& Behavior, 7: 1456-1466.

17. Heidari, P. and H. Najafi Zarrini. 2016. Classification and gene expression analysis of bzip family in tomato root under sub-optimal temperature. Journal of Crop Breeding, 8(17): 17-23 (In Persian).

18. Hiremath, P.J., A. Farmer, S.B. Cannon, J. Woodward, H. Kudapa and R. Tuteja. 2011. Large-scale transcriptome analysis in chickpea (Cicer arietinum L.), an orphan legume crop of the semi-arid tropics of Asia and Africa. Plant Biotechnology Journal, 9: 922-931.

19.Huang, G.T., S.L. Ma, L.P. Bai, L. Zhang, H. Ma and P. Jia. 2012. Signal transduction during cold, salt, and drought stresses in plants. Molecular Biology Reports, 39: 969-987.

20.Jaganathan, D., M. Thudi, S. Kale, S. Azam, M. Roorkiwal and P.M. Gaur. 2015. Genotyping-bysequencing based intra-specific genetic map refines a "QTL-hotspot" region for drought tolerance in chickpea. Molecular Genetics and Genomics, 290: 559-571.

21.Jin, J., H. Zhang, L. Kong, G. GAO and J. Luo. 2013. Plant TFDB 3.0: a portal for the functional and evolutionary study of plant transcription factors. Nucleic Acids Research, 42: 1182-D1187.

22.Jukanti, A.K., P.M. Gaur, C. Gowda and R.N. Chibbar. 2012. Nutritional quality and health benefits of chickpea (Cicer arietinum L.): a review. British Journal of Nutrition, 108: S11-S26.

23. Kale, S.M., D. Jaganathan, P. Ruperao, C. Chen, R. Punna and H. Kudapa. 2015. Prioritization of candidate genes in "QTL-hotspot" region for drought tolerance in chickpea (Cicer arietinum L.). Scientific Reports, 5: 15296.

24. Kashiwagi, J., L. Krishnamurthy, J.H. Crouch and R. Serraj. 2006. Variability of root length density and its contributions to seed yield in chickpea (Cicer arietinum L.) under terminal drought stress. Field Crops Research, 95: 171-181.

25.Liu, J.H., T. Peng and W. Dai. 2014. Critical cis-acting elements and interacting transcription factors: key players associated with abiotic stress responses in plants. Plant Molecular Biology Reporter, 32: 303-317.

26.Liu, L., Z. Zhang, J. Dong and T. Wang. 2016. Overexpression of MtWRKY76 increases both salt and drought tolerance in Medicago truncatula. Environmental and Experimental Botany, 123: 50-58.

27.Liu, Y., X. Yu, S. Liu, H. Peng, A. Mijiti and Z. Wang. 2017. A chickpea NAC-type transcription factor, CarNAC6, confers enhanced dehydration tolerance in Arabidopsis. Plant Molecular Biology Reporter, 35: 83-96.

28.Lobell, D.B., W. Schlenker and J. Costa-Roberts. 2011. Climate trends and global crop production since 1980. Science, 333: 616-620.

29. Mansourifar, C., M. Shaban, M. Ghobadi and A.R. Ajirlu. 2011. Effect of drought stress and N fertilizer on yield, yield components and grain storage proteins in chickpea (Cicer arietinum L.) cultivars. African Journal of Plant Science, 5: 634-642.

30. Moucheshi, S., B. Heidari and E. Farshadfar. 2009. Evaluation of stress indices for drought tolerance screening of chickpea (Cicer arietinum L.). Journal of Crop Breeding, 1 (4): 49-64 (In Persian).

31. Nakashima, K., H. Takasaki, J. Mizoi, K. Shinozaki and K. Yamaguchi-Shinozaki. 2012. NAC transcription factors in plant abiotic stress responses. Biochimica ET Biophysica Acta, 1819: 97-103.

32. Nakashima, K., L.S.P. Tran, D. Van Nguyen, M. Fujita, K. Maruyama and D. Todaka. 2007. Functional analysis of a NAC-type transcription factor OsNAC6 involved in abiotic and biotic stress-responsive gene expression in rice. The Plant Journal, 51: 617-630.

33. Nguyen, K.H., C.V. Ha, Y. Watanabe, U.T. Tran, M. Nasr Esfahani and D.V. Nguyen. 2015. Correlation between differential drought tolerability of two contrasting drought-responsive chickpea cultivars and differential expression of a subset of CaNAC genes under normal and dehydration conditions. Frontiers in Plant Science, 6: 449.

34.Ngwe, T., Y. Nukui, S. Oyaizu, G. Takamoto, S. Koike, K. Ueda. 2012. Bean husks as a supplemental fiber for ruminants: potential use for activation of fibrolytic rumen bacteria to improve main forage digestion. Animal Science Journal, 83: 43-49.

35.Pérez-Clemente, R.M., V. Vives, S.I. Zandalinas, M.F. López-Climent, V. Muñoz and A. GómezCadenas. 2012. Biotechnological approaches to study plant responses to stress. BioMed Research International, 13-20.

36. Rubio, L.A. 2005. Ileal digestibility of defatted soybean, lupin and chickpea seed meals in cannulated Iberian pigs: I. Proteins. Journal of the Science of Food and Agriculture, 85: 1313-1321. 
$1 f$. ارزيابى عوامل رونويسى در يك مطالعه ترانسكرييتوم در نخود .

38. Schaller, G.E., J.J. Kieber and S.H. Shiu. 2008. Two-component signaling elements and histidylaspartyl phosphorelays. The Arabidopsis Book, 112 pp.

39. Shao, H.B., L.Y. Chu, C.A. Jaleel, P. Manivannan, R. Panneerselvam and M.A. Shao. 2009. Understanding water deficit stress-induced changes in the basic metabolism of higher plantsbiotechnologically and sustainably improving agriculture and the eco-environment in arid regions of the globe. Critical Reviews in Biotechnology, 29: 131-151.

40. Singh, D. and A. Laxmi. 2015. Transcriptional regulation of drought response: a tortuous network of transcriptional factors. Frontiers in Plant Science, 6 pp.

41. Singh, K.B., R.C. Foley and L. Oñate-Sánchez. 2002. Transcription factors in plant defense and stress responses. Current Opinion in Plant Biology, 5: 430-436.

42.Singh, R.P., S. Rizvi and P.K. Jaiwal. 2003. Genetic engineering for enhancing abiotic stress tolerance. Improvement Strategies of Leguminosae Biotechnology. Springer, 223-243.

43.Su, L.T., J.W. Li, D.Q. Liu, Y. Zhai, H.J. Zhang and X.W. Li. 2014. A novel MYB transcription factor, GmMYBJ1, from soybean confers drought and cold tolerance in Arabidopsis thaliana. Gene, 538: 46-55.

44.Thudi, M., H.D. Upadhyaya, A. Rathore, P.M. Gaur, L. Krishnamurthy and M. Roorkiwal. 2014. Genetic dissection of drought and heat tolerance in chickpea through genome-wide and candidate gene-based association mapping approaches. PLoS One, 9: 96758.

45.Varshney, R.K., C. Song, R.K. Saxena, S. Azam, S. Yu and A.G. Sharpe. 2013. Draft genome sequence of chickpea (Cicer arietinum) provides a resource for trait improvement. Nature Biotechnology, 31: 240-246.

46. Varshney, R.K., M. Thudi, S.N. Nayak, P.M. Gaur, J. Kashiwagi and L. Krishnamurthy. 2014. Genetic dissection of drought tolerance in chickpea (Cicer arietinum L.). TAG. Theoretical and Applied Genetics, 127: 445-462.

47.Wilkinson, S. and W.J. Davies. 2010. Drought, ozone, ABA and ethylene: new insights from cell to plant to community. Plant, Cell \& Environment, 33: 510-525.

48. Yu, X., Y. Liu, S. Wang, Y. Tao, Z. Wang and Y. Shu. 2016. CarNAC4, a NAC-type chickpea transcription factor conferring enhanced drought and salt stress tolerances in Arabidopsis. Plant Cell Reports, 35: 613-627.

49. United Nations. 2015. The World Population Prospects. New York, NY: United Nations Department of Economic and Social Affairs. 


\title{
Transcription Factors Evaluation in a Transcriptome Analysis on Chickpea (Cicer arietinum L.) Under Drought Stress
}

\section{Keyvan Mahdavi Mashaki ${ }^{1,6}$, Ali Asghar Nasrollahnezhad Ghomi ${ }^{2}$, Khalil Zaynali Nezhad $^{3}$, Ahad Yamchi ${ }^{3}$, Hasan Soltanloo ${ }^{4}$, Mahendar Thudi ${ }^{5}$ and Rajeev Kumar Varshney ${ }^{5}$}

\author{
1, 3 and 4- Graduated PhD Student, Assistant Professor and Associate Professor, Gorgan University of Agricultural \\ Sciences and Natural Resources \\ 2- Assistant Professor, Gorgan University of Agricultural Sciences and Natural Resources, (Corresponding \\ author: Ali1346nn@yahoo.com) \\ 5- Professors, International Crops Research Institute for the Semi-Arid Tropics (ICRISAT), Hyderabad, India \\ 6- Assistant Professor of the Rice Research Institute of Iran, Mazandaran Branch, Agricultural Research, Education \\ and Extension Organization (AREEO), Amol, Iran \\ Received: July 2, $2017 \quad$ Accepted: September 12, 2017
}

\begin{abstract}
Drought causes detrimental effect on growth and productivity of many plants, including crops. Chickpea (Cicer arietinum L.) as one of the most important legume crops is subjected to terminal drought stress in arid and semi-arid regions. Transcription factors (TFs) play key roles during signal transduction and adaptation response to abiotic stresses such as drought. In the present study, TFs were assessed in a transcriptome analysis in the root and the shoot tissues of two contrasting drought responsive kabuli chickpea. Out of 4572 differentially expressed genes, 1806 TFs were identified using search on the plant transcription factor database (PTFD). The highest members (101) of the TFs belonged to bHLH family, followed by ERF (87), kinase superfamily (76), NAC (74), MYB (72), WRKY (72), etc. The comparison of the tolerant (Bivanij) and the sensitive (Hashem) cultivars under drought stress showed that the TFs were differently distributed based on the cultivars and the tissue types. The TF families including B3, NAC, MYB, WRKY, bHLH, etc. had most members in response to the drought stress. Furthermore, the results revealed that several TFs which were involved in abiotic stress-related responses and major biosynthetic pathways such as $\mathrm{ABA}$ and proline biosynthesis were upregulated in the shoot of Bivanij as compared to Hashem indicating the vital role of the shoot for inducing drought tolerance in the tolerant cultivar. As result, these findings help the researches to better understanding of signal transduction and stress-related regulating networks in chickpea and provide the transferring of key TFs and promoting drought tolerance by genetic engineering.
\end{abstract}

Keywords: Chickpea, Drought Stress, Signal Transduction, Transcription Factors, Transcriptome 